\title{
Metabolic profiling of isolated mitochondria and cytoplasm reveals compartment-specific metabolic responses
}

\author{
Daqiang Pan ${ }^{1,2} \cdot$ Caroline Lindau $^{3,4} \cdot$ Simon Lagies ${ }^{1,4,5} \cdot$ Nils Wiedemann $^{3,6} \cdot$ Bernd Kammerer $^{1,6}$
}

Received: 5 December 2017 / Accepted: 20 March 2018 / Published online: 31 March 2018

(c) The Author(s) 2018

\begin{abstract}
Introduction Subcellular compartmentalization enables eukaryotic cells to carry out different reactions at the same time, resulting in different metabolite pools in the subcellular compartments. Thus, mutations affecting the mitochondrial energy metabolism could cause different metabolic alterations in mitochondria compared to the cytoplasm. Given that the metabolite pool in the cytosol is larger than that of other subcellular compartments, metabolic profiling of total cells could miss these compartment-specific metabolic alterations.

Objectives To reveal compartment-specific metabolic differences, mitochondria and the cytoplasmic fraction of baker's yeast Saccharomyces cerevisiae were isolated and subjected to metabolic profiling.

Methods Mitochondria were isolated through differential centrifugation and were analyzed together with the remaining cytoplasm by gas chromatography-mass spectrometry (GC-MS) based metabolic profiling.

Results Seventy-two metabolites were identified, of which eight were found exclusively in mitochondria and sixteen exclusively in the cytoplasm. Based on the metabolic signature of mitochondria and of the cytoplasm, mutants of the succinate dehydrogenase (respiratory chain complex II) and of the $\mathrm{F}_{\mathrm{O}} \mathrm{F}_{1}$-ATP-synthase (complex V) can be discriminated in both compartments by principal component analysis from wild-type and each other. These mitochondrial oxidative phosphorylation machinery mutants altered not only citric acid cycle related metabolites but also amino acids, fatty acids, purine and pyrimidine intermediates and others.

Conclusion By applying metabolomics to isolated mitochondria and the corresponding cytoplasm, compartment-specific metabolic signatures can be identified. This subcellular metabolomics analysis is a powerful tool to study the molecular mechanism of compartment-specific metabolic homeostasis in response to mutations affecting the mitochondrial metabolism.
\end{abstract}

Keywords ATP-synthase $\cdot$ Metabolomics $\cdot$ Metabolic phenotyping $\cdot$ Mitochondria $\cdot$ Succinate dehydrogenase $\cdot$ Yeast

Electronic supplementary material The online version of this article (https://doi.org/10.1007/s11306-018-1352-x) contains supplementary material, which is available to authorized users.

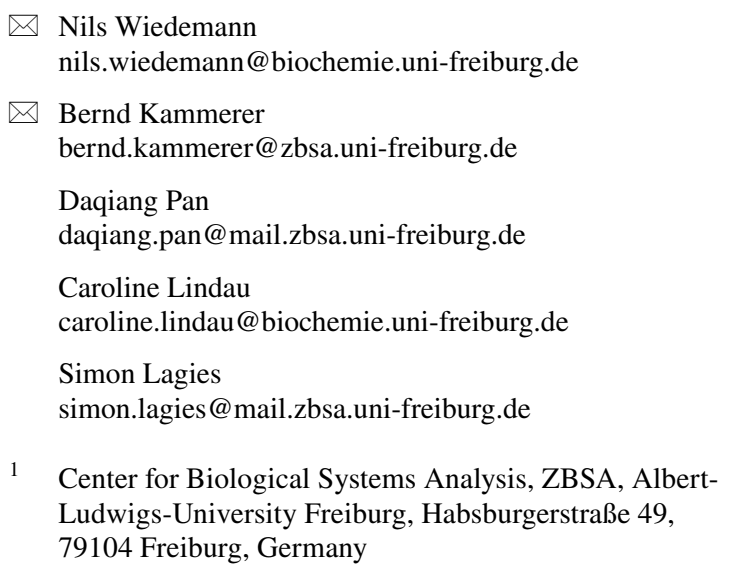

2 Institute of Pharmaceutical Sciences, Albert-Ludwigs-University Freiburg, 79104 Freiburg, Germany

3 Institute of Biochemistry and Molecular Biology, ZBMZ, Faculty of Medicine, Albert-Ludwigs-University Freiburg, Stefan-Meier-Str. 17, 79104 Freiburg, Germany

4 Faculty of Biology, University of Freiburg, 79104 Freiburg, Germany

5 Spemann Graduate School of Biology and Medicine (SGBM), Albert-Ludwigs-University Freiburg, 79104 Freiburg, Germany

6 BIOSS Centre for Biological Signalling Studies, University of Freiburg, 79104 Freiburg, Germany 


\section{Introduction}

Mitochondria have been described as the power houses of the cell due to their major function in adenosine triphosphate (ATP) synthesis, which is mainly based on the pyruvate dehydrogenase, the $\beta$-oxidation of fatty acids, the citric acid cycle (TCA cycle), and the respiratory chain (RC) (Nunnari and Suomalainen 2012; van der Bliek et al. 2017). Defects in mitochondria are linked to human diseases such as mitochondrial encephalomyopathy, Alzheimer's disease, diabetes and cancer (Schapira 2012; Gaude and Frezza 2014; Paglia et al. 2016). Therefore, it is crucial to identify mechanisms of mitochondrial diseases so that possible therapies can be developed.

Mass spectrometry (MS) based metabolomics has become a promising research tool for various biological systems, including bacteria, plants, mammalian cells, tissues and body fluids (Fiehn et al. 2000; Liesenfeld et al. 2013; Sana et al. 2013; Willmann et al. 2016). Genetic and other pathological or environmental changes can result in alterations of metabolites, which can be revealed by metabolomics. Alterations of metabolites have been reported in blood plasma, urine of cancer patients and cancer cell lines, providing promising possibilities of biomarker discoveries (Bullinger et al. 2007; Frickenschmidt et al. 2008; Asiago et al. 2010; Shen et al. 2013; Armitage and Barbas 2014; Willmann et al. 2015). Additionally, metabolomics helps in understanding cellular mechanisms by showing the metabolic status of drug-treated or nontreated cancer cells (Jain et al. 2012; Budczies et al. 2015; Pan et al. 2016). Since metabolomics has been described as the link between genotype and phenotype (Fiehn 2002), Stefely et al. (2016) have profiled 174 yeast strains with different single gene knockouts to elucidate the mitochondrial protein functions. However, whole cells were applied in the profiling, which could not uncover the compartmentspecific metabolic signature. In contrast, van Vranken and Rutter (2016) valued subcellular compartment metabolic profiling by coining the term 'the whole (cell) is less than the sum of its parts'.

Compartment-specific mitochondrial metabolite levels were already analyzed by Siess et al. (1978) for TCA cycle related metabolites and by Ross-Inta et al. (2008) for mitochondrial amino acids. Although isolation of mitochondria has been established and optimized in several research groups (Meisinger et al. 2006; Boldogh and Pon 2007; Fernández-Vizarra et al. 2010), mitochondrial metabolomics using modern MS equipment has not been applied widely due to technical difficulties. One of the first studies analyzing isolated mitochondria by state of the art metabolomics methods was published by Roede et al. (2012). They could distinguish male from female and wild type from mutant. This was the first time that phenotypes could be discriminated from each other based on the mitochondrial metabolic signature. Although only part of the detected features could be annotated as metabolites based on $\mathrm{m} / \mathrm{z}$ and retention time (RT), the remaining features indicated a large amount of unidentified known and/ or unknown metabolites in mitochondria. A compartmentspecific metabolomics study was conducted by filtration of Chinese hamster ovary cells and revealed a very low ATP pool within mitochondria (Matuszczyk et al. 2015). Recently, Chen et al. (2016) determined absolute concentrations for over 100 mitochondrial matrix metabolites in comparison to whole cells employing immunopurification of mitochondria using an artificial epitope tag fused to an outer membrane anchor. In this study a higher concentration was observed for most of the metabolites in whole cells compared to mitochondria. Besides that, the metabolic responses to $\mathrm{RC}$ inhibitors were in mitochondria stronger compared to whole cells. Hence, it is reasonable to expect that metabolic alterations are compartmentspecific. By means of our approach compartment-specific metabolic signatures could be detected, which can be related to specific metabolic alterations in mitochondrial mutants.

\section{Materials and methods}

\subsection{Yeast}

Saccharomyces cerevisiae strains atp $4 \Delta$ (4333), sdh $2 \Delta$ (1996) and the corresponding wildtype (WT) BY4741 (1354) with the genotype MATa, ura $3 \Delta 0$, leu $2 \Delta 0$, his $3 \Delta 1$, met15 $\Delta 0$ (Baker Brachmann et al. 1998) were obtained from Euroscarf. Yeast cells were cultured in liquid synthetic galactose media $(0.67 \%$ [w/v] yeast nitrogen base without amino acids (Becton, Dickinson and Company, MD, USA), $0.77 \mathrm{~g} / \mathrm{L}$ SC amino acids (MP Biomedicals, USA), $2 \%$ [w/v] galactose (Sigma-Aldrich)) at $30{ }^{\circ} \mathrm{C}$ under shaking at $130 \mathrm{rpm}$. Cells were harvested in exponential phase.

\subsection{Isolation of mitochondria and the cytoplasm}

Based on the protocol from Meisinger et al. (2006) and the isolation buffer used by Corcelli et al. (2010), mitochondria were isolated with optimized MS compatible KClEM isolation buffer [180 $\mathrm{mM} \mathrm{KCl}, 1 \mathrm{mM}$ EDTA and $5 \mathrm{mM}$ MOPS-KOH ( $\mathrm{pH}$ 7.2)]. Briefly, after incubation in dithiothreitol buffer, yeast cells were washed with KClEM buffer and treated with zymolyase (Seigaku), subsequently washed and pottered in ice-cold KClEM buffer. The nuclei were pelleted by subsequently increasing the centrifugal force $250 \times g$ for $2 \mathrm{~min}, 1000 \times g$ for $4 \mathrm{~min}$ and $1900 \times g$ for $4 \mathrm{~min}$ 
in a single centrifugation run. The post nuclear supernatant was subjected to a $15 \min 16,800 \times g$ centrifugation step to pellet the mitochondrial fraction. The post mitochondrial supernatant was collected and regarded as remaining cellular cytoplasm for further analysis. The protein concentration of isolated mitochondria was determined using the Bradford assay (Roth). Mitochondria were aliquoted into $1 \mathrm{mg}$ aliquots and the corresponding fraction of cytoplasm was calculated based on the quantity of mitochondria and the volume of the cytoplasmic fraction. To test the integrity of the isolated mitochondria, $30 \mu \mathrm{g}$ mitochondria were thawed on ice and treated with an indicated amount of Proteinase $\mathrm{K}$ (Roche) for $10 \mathrm{~min}$ on ice. Proteinase $\mathrm{K}$ was inactivated by addition of $2.4 \mathrm{mM}$ PMSF (Phenylmethylsulfonylfluorid, Roth) and further incubated on ice for $10 \mathrm{~min}$. Mitochondria were pelleted for $10 \mathrm{~min}$ at $20,817 \times g(14,000 \mathrm{rpm}, 5417 \mathrm{R}$, Eppendorf) at $4{ }^{\circ} \mathrm{C}$ and washed with $200 \mu \mathrm{L}$ isolation buffer containing $1 \mathrm{mM}$ PMSF.

\subsection{SDS-PAGE and western blotting}

For SDS-PAGE analysis, mitochondria were resuspended in Laemmli buffer (60 mM Tris (MP Biomedicals), $\mathrm{pH} 6.8$, $2 \%$ [w/v] SDS (SDS, Serva), 10\% [v/v] glycerol (Honeywell Research Chemicals), 0.02\% [w/v] bromphenol blue (Sigma)) containing 1-2 mM PMSF and 1\% [v/v] 2-mercaptoethanol and incubated for $15 \mathrm{~min}$ at $60{ }^{\circ} \mathrm{C}$ with shaking at $1400 \mathrm{rpm}$. Samples were analyzed by using $10 \%$ Tris-Tricine polyacrylamide gels containing $10 \%$ polyacrylamide (49.5\% [w/v] acrylamide (Roth), 3\% [w/v] bisacrylamide (Serva)). Gel electrophoresis was performed in anode ( $0.2 \mathrm{M}$ Tris/HCl, $\mathrm{pH}$ 8.9) and cathode (0.1 M Tris, $0.1 \mathrm{M}$ Tricine (Roth), $0.1 \%$ [w/v] dodecylsulfate-Na-salt (SDS, Serva), $\mathrm{pH}$ 8.25 ) buffer at $70 \mathrm{~mA}, 600 \mathrm{~V}$ for $3.5 \mathrm{~h}$. Afterwards, proteins were transferred to PVDF membranes (Immobilon-P, Millipore) by performing a standard semi-dry western blot transfer at $250 \mathrm{~mA}, 25 \mathrm{~V}$ for $2.5 \mathrm{~h}$ in western transfer buffer (20 mM Tris, $150 \mathrm{mM}$ glycine (MP Biomedicals), 0.02\% [w/v] SDS, 20\% [v/v] methanol). Membranes were stained in $0.2 \%$ Coomassie R250 (Roth), 10\% [v/v] acetic acid (Roth) and $30 \%$ [v/v] ethanol and subsequently destained in $100 \%$ methanol and washed shortly in TBST [200 mM Tris/HCl, $\mathrm{pH} 7.5,1.25 \mathrm{M} \mathrm{CaCl}_{2}, 0.1 \%$ Tween20 (Sigma)] before they were incubated for $1 \mathrm{~h}$ in $5 \%$ [w/v] fat-free dried milk powder (Frema Reform) in TBST at room temperature. Membranes were shortly washed in TBST and then incubated for $3 \mathrm{~h}$ in the indicated primary antibodies at room temperature. After three 5 min washing steps in TBST, the membranes were incubated for $1 \mathrm{~h}$ at room temperature in secondary anti-rabbit IgG antibody (Sigma), diluted 1:5000 in 5\% [w/v] fat-free dried milk powder in TBST, which is coupled to a horseradish peroxidase. After three 5 min washing steps in TBST, membranes were incubated in ECL solution (Haan and Behrmann 2007) and chemiluminescence signals were detected by a LAS-4000 camera system (Fujifilm). Antibodies used in this study are listed by antigen, dilution and number: Tom70, 1:500 TBST + 5\% milk, GR657-4; Tom40, 1:500 TBST $+5 \%$ milk, GR168-4; Tim44, 1:400 TBST +5\% milk, GR127-6; Pam17, 1:500 TBST + 5\% milk, GR3885-5.

\subsection{Metabolite extraction, GC-MS analysis and data evaluation}

Mitochondrial pellets corresponding to $1 \mathrm{mg}$ protein and half of the volume of each corresponding cytoplasm were applied to metabolic profiling in technical triplicate. The results were reproduced by analyzing different biological preparations. The cytoplasm was dried in a Concentrator plus vacuum rotator (Eppendorf, Germany) before $1 \mathrm{~mL}$ extraction medium $\left(-20{ }^{\circ} \mathrm{C}\right.$ methanol/water (v/v: $\left.90 / 10\right)$ containing $1 \mu \mathrm{g} / \mathrm{mL}$ of $\beta$-phenylglucose and ribitol as internal standards) was added to each mitochondrial or cytoplasmic pellet. Samples were incubated at $4{ }^{\circ} \mathrm{C}$ and $1200 \mathrm{rpm}$ for $10 \mathrm{~min}$ after short vortex. After centrifugation at $21,000 \times \mathrm{g}$ and $4{ }^{\circ} \mathrm{C}$ for $10 \mathrm{~min}$, the metabolite-containing supernatants were transferred to new reaction tubes and dried in a Concentrator plus vacuum rotator and stored under nitrogen atmosphere at $-80^{\circ} \mathrm{C}$ until derivatization.

Based on the protocol of Fiehn (2006), sample derivatization, gas chromatography-mass spectrometry (GC-MS) analysis and data evaluation were performed as described by Pan et al. (2016). Briefly, the prewarmed pellets were derivatized by shaking with $20 \mu \mathrm{L} 20 \mathrm{mg} / \mathrm{mL}$ methoxyamine hydrochloride (Sigma-Aldrich, Germany) in pyridine (Sigma-Aldrich, Germany) at $1200 \mathrm{rpm}$ and $28{ }^{\circ} \mathrm{C}$ for $90 \mathrm{~min}$ and, after short centrifugation, with $50 \mu \mathrm{L} \mathrm{N}$-methyl$\mathrm{N}$-trimethylsilyltrifluoroacetamide (Sigma-Aldrich, Germany) at $1200 \mathrm{rpm}$ and $37^{\circ} \mathrm{C}$ for $30 \mathrm{~min}$.

An HP-5MS capillary column (Agilent, Germany) with the dimension of $60 \mathrm{~m} \times 0.25 \mathrm{~mm} \times 0.25 \mu \mathrm{m}$ was used for GC separation with helium as carrier gas. The oven temperature was held at $80{ }^{\circ} \mathrm{C}$ for the first 2 min, then increased to $320{ }^{\circ} \mathrm{C}$ within $50 \mathrm{~min}$ and kept at $320^{\circ} \mathrm{C}$ for $10 \mathrm{~min}$. A C10-C40 $n$-alkane standard mixture (Neochema, Bodenheim, Germany) was applied at the beginning for retention index calculation. All GC-MS data files were processed with AMDIS (Version 2.71, National Institute of Standards and Technology, Gaithersburg, MD, USA) and the web-based tool SpectConnect (Styczynski et al. 2007) to perform the peak picking, spectral deconvolution and compound identification. Annotation of metabolites was performed according to the retention index deviation $(<5 \%)$ and spectra match score $(>750)$ in NIST (Version 2.2, National Institute of Standards and Technology, Gaithersburg, MD, USA), GolmDB (Kopka et al. 2005) and Fiehnlib (Kind et al. 2009) databases. Annotated metabolites were normalized by 
internal standard and protein amount. Finally, MetaboAnalyst 3.0 (Xia et al. 2015) was used for statistical analysis.

\section{Results and discussion}

\subsection{Inner membrane integrity of isolated mitochondria in mass spectrometry compatible potassium chloride buffer}

Since $S$. cerevisiae has been used as a system for modeling mitochondrial diseases (Goffeau et al. 1996; Herrgard et al. 2008; Lasserre et al. 2015), baker's yeast was selected to analyze the compartment-specific metabolic alteration of mutants affecting the mitochondrial metabolism. In addition to isolated mitochondria of wild type (WT) and RC mutants, the corresponding cytoplasm was also analyzed by GC-MS based metabolic profiling. Yeast cells were cultivated in galactose containing medium, inducing a respiratory growth behavior in the strain BY4741 (Morgenstern et al. 2017). Chen et al. (2016) has described the incompatibility of traditional mitochondrial isolation buffers with liquid chromatography-MS and applied a potassium chloride based isolation buffer. Along with their findings, sugars as osmolytes used in the traditional mitochondrial isolation buffers were also found in our approach to be incompatible with GC-MS. We established a mitochondria isolation procedure by differential centrifugation using the potassium chloride buffer established by Corcelli et al. (2010) to achieve a direct comparison of mitochondrial and cytoplasmic metabolites. To avoid unnecessary equilibration of metabolites across the mitochondrial membrane channels and transporters, we minimized the isolation time after disruption of the cells to only two centrifugation steps: first to pellet the nuclei by centrifugation for a total of $10 \mathrm{~min}$ and second to pellet mitochondria for $15 \mathrm{~min}$. The post-mitochondrial supernatant was collected as corresponding cytoplasm consisting

A

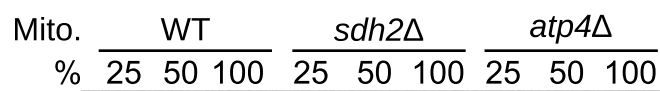

Tom70 -

Tom40 -
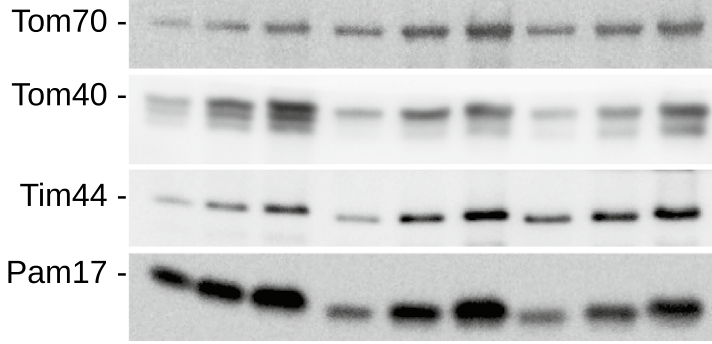

Fig. 1 Integrity of the mitochondrial inner membrane. a Mitochondrial protein steady state levels were analyzed by SDS-PAGE and western blotting with antibodies directed against the indicated pro- of the cytosol and the other remaining organelles. The mitochondrial and cytoplasmic fractions were then used to detect compartment-specific metabolic signatures. To prove the feasibility of our approach we selected two classical mitochondrial mutants: The electron transport chain and TCA cycle mutant strain with a deletion of the succinate dehydrogenase (complex II) subunit $2(s d h 2 \Delta)$ and the $\mathrm{F}_{\mathrm{O}} \mathrm{F}_{1}$-ATP synthase (complex V) subunit 4 (atp4 4 ) deleted strain. Mitochondrial proteins were subjected to SDS-PAGE analysis and subsequent western blotting, indicating similar levels of translocase of the outer mitochondrial membrane (TOM) proteins Tom70 and Tom 40 and translocase of the inner mitochondrial membrane (TIM) protein Tim44 and of the presequence translocase associated import motor (PAM) protein Pam17 (Wiedemann and Pfanner 2017) (Fig. 1a). To test the integrity of the mitochondrial inner membrane upon isolation in MS compatible potassium chloride buffer, isolated mitochondria were treated with increasing concentrations of Proteinase K (Prot. K). The protease was inactivated by phenylmethylsulfonyl fluoride before mitochondrial proteins were analyzed by SDS-PAGE and western blotting. The outer membrane protein Tom70, which contains a large cytosolic domain exposed to the outside of mitochondria, is efficiently degraded by addition of the external protease. In contrast, the mitochondrial matrix protein Tim44 remains largely unaffected by protease treatment, indicating that the mitochondrial inner membrane remains intact (Fig. 1b).

\subsection{Compartment-specific metabolic signature in mitochondria and cytoplasm}

Isolated mitochondria and the corresponding cytoplasm of WT were analyzed concomitantly by GC-MS based metabolic profiling. Seventy-two metabolites were identified altogether, of which eight were found exclusively in mitochondria while sixteen were found exclusively in the cytoplasm, indicating compartment-specific distribution of

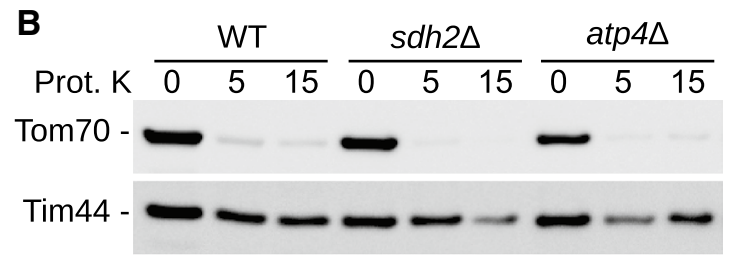

teins. b Isolated mitochondria suspended in potassium chloride based isolation buffer were treated with Proteinase K (Prot. K) and analyzed as described in panel (a) 


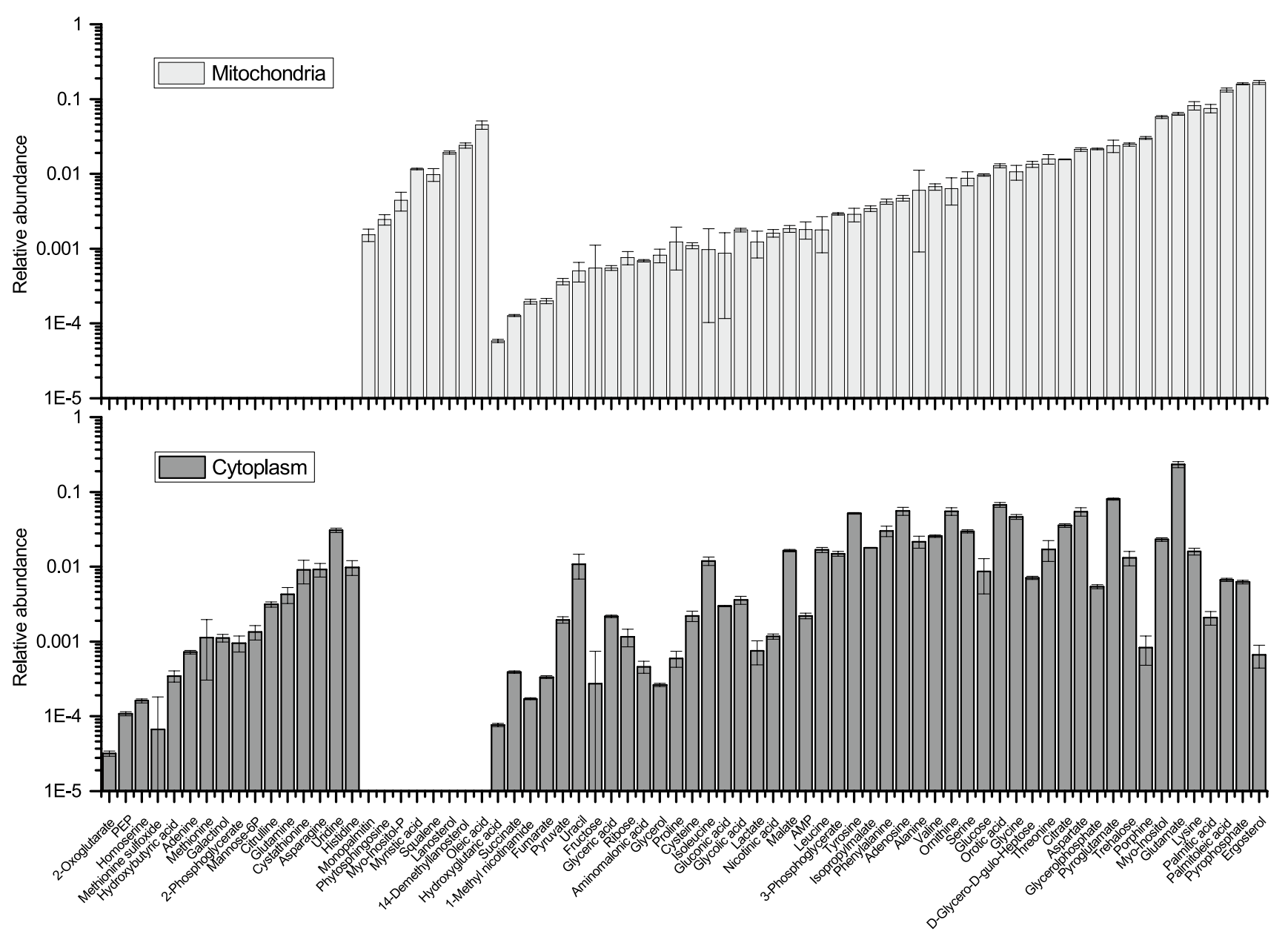

Fig. 2 Bar charts indicating the relative abundance of identified metabolites in mitochondria and the cytoplasm of wild type (mean $\pm \mathrm{SD}, \mathrm{n}=3$ ). Eight metabolites were found exclusively in mito- chondria and sixteen exclusively in the corresponding cytoplasm. $P E P$ phosphoenolpyruvate, Mannose-6P mannose-6-phophate, MyoInositol-P myo-inositol-phosphate, $A M P$ adenosine monophosphate metabolites in our preparations (Fig. 2). These findings are in good agreement with the mitochondrial isolation procedure by immunopurification (Chen et al. 2016). This indicates that within the time of the mitochondrial isolation, compartment-specific metabolite distribution was preserved. The mitochondria specific metabolites are mainly fatty acids and steroid intermediates. Given that many metabolites show higher levels in the cytoplasmic fraction compared to mitochondria (Chen et al. 2016), it is possible that metabolic alterations of mutants, affecting the mitochondrial metabolism, may be buried in the metabolite pool of total cells.

\subsection{Mutations induce compartment-specific metabolic regulation}

To analyze if compartment-specific metabolic responses, caused by changes of the mitochondrial metabolism, can be detected, mitochondria and the corresponding cytoplasmic fractions of WT, atp $4 \Delta$ and $s d h 2 \Delta$ yeast cells were subjected to GC-MS. Citrate synthase is used to normalize mitochondrial data in many studies (Mogensen et al. 2007; Chen et al. 2016). However, Morgenstern et al. (2017) showed that the level of citrate synthase 1 (Cit1) is variable depending on the growth conditions. The normalization using citrate synthase would be problematic given that ATP4 and $S D H 2$ are functionally connected to the TCA cycle and their deletions will impact the growth conditions, the level of mitochondrial citrate synthase (Cit1) and its activity. Therefore, the GC-MS data was normalized, in addition to internal standard, by the protein content of the mitochondrial fraction, whose amount was verified by employing specific antibodies against four selected mitochondrial marker proteins Tom70, Tom40, Tim44 and Pam17. The fold change (FC) of metabolites in mutants compared to WT is regarded as the metabolic response to the mutation of a specific gene. In order to find significantly altered metabolites, analysis of variance (ANOVA) was performed, followed by false discovery rate (FDR) to exclude the false 


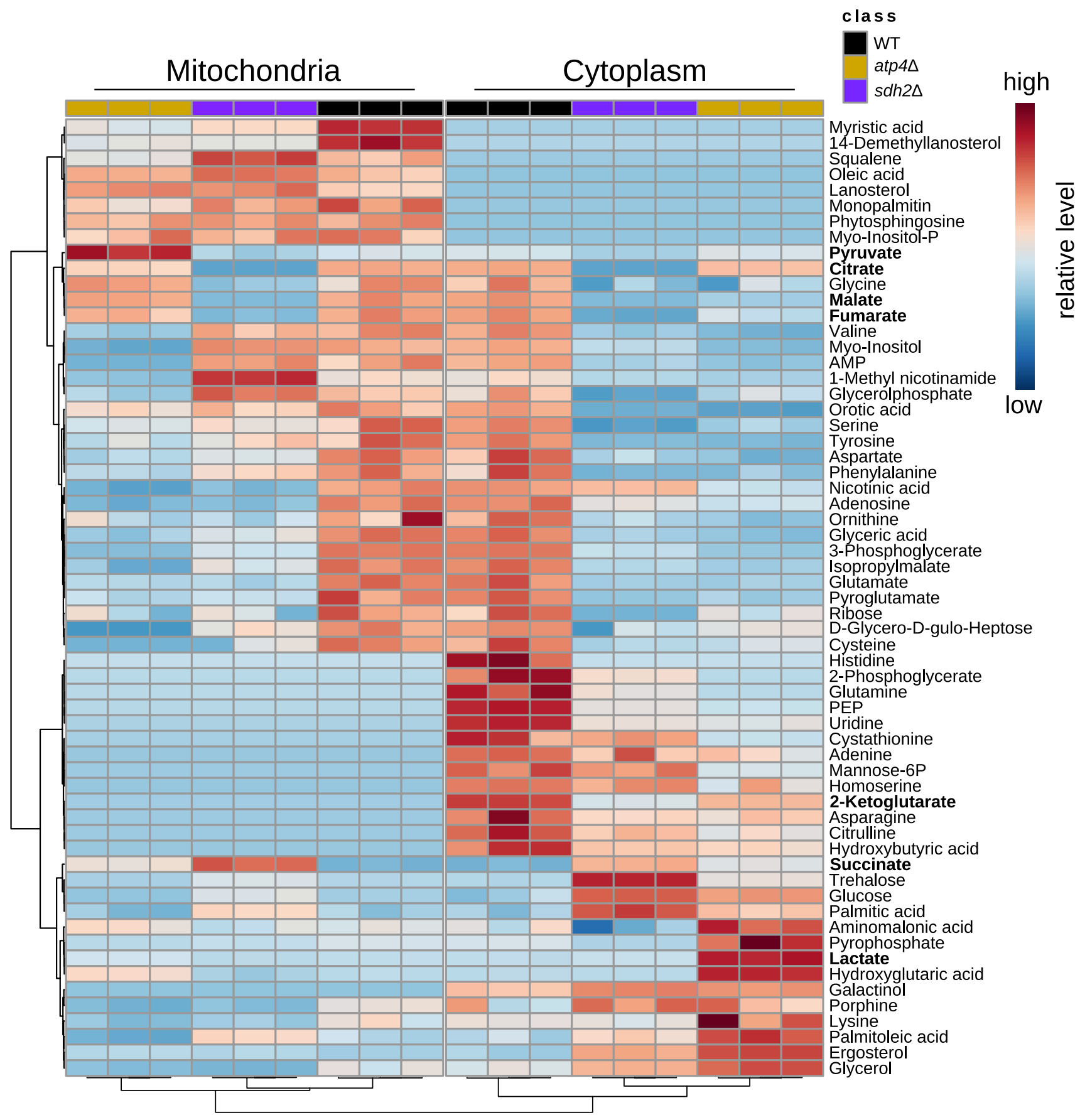

Fig. 3 Heatmap shows the fold changes of metabolites in mitochondria and the cytoplasm of WT and the two mutants, atp $4 \Delta$ and $s d h 2 \Delta$. Lactate, pyruvate and detected TCA cycle intermediates are

significant metabolites. Sixty-one metabolites showed significantly ( $<<0.001, \mathrm{q}<0.001)$ altered FCs (see Supplementary Table S1). Based on these FCs, a heatmap (Fig. 3) was generated, presenting the change of each metabolite in mitochondria and the cytoplasm of atp $4 \Delta$ and $s d h 2 \Delta$ compared to WT. Consequently, the alteration of metabolites in atp $4 \Delta$ and $s d h 2 \Delta$ are opposing for a large fraction of the highlighted in bold text. Only 61 out of 72 identified metabolites are shown here with a q value $<0.001$

detected metabolites between mitochondria and the cytoplasm (Fig. S1), indicating different metabolic activities in both compartments. Moreover, both mutants show a specific metabolite alteration pattern. As a result of these mutations affecting mitochondrial respiration, not only the TCA cycle, but also amino acid metabolism, fatty acid metabolism, glycolysis and nucleotide metabolism were affected. 
Based on the detected metabolites principal component analysis (PCA) can discriminate both the mitochondrial and the cytoplasmic compartments from each other. As shown in Fig. 4a, PC2 discriminates mitochondria from the cytoplasm. Based on the metabolic alterations caused by the deletion of ATP4 or SDH2, PC3 discriminates atp $4 \Delta$ and $s d h 2 \Delta$ from each other and from WT. The loading map with the values for PC2 and PC3 in Fig. 4b shows how single metabolites influenced the discrimination of compartments and strains. Consequently, the compartment-specific metabolites (negative PC2 value for mitochondria-specific and positive for cytoplasm-specific) as observed in Fig. 2 contributed to the discrimination in PC2, while TCA cycle related intermediates like pyruvate, citrate, fumarate and malate made a major contribution to $\mathrm{PC} 3$, highlighting their dysregulation in the mutant mitochondria. Besides that, they are also clustered together in the heatmap, displaying a similar regulation pattern.

Using this compartment-specific metabolomics approach we identified a number of metabolites, which show a differential regulation pattern between mitochondria and the cytoplasm (Fig. 5). The levels of amino acids like glutamate, ornithine, phenylalanine, aspartate and serine are reduced in both compartments of atp $4 \Delta$ and $s d h 2 \Delta$. However, proline showed significantly reduced levels in the cytoplasm and relatively normal mitochondrial levels compared to WT. In contrast to the generally reduced amino acids, the levels of alanine are slightly decreased in the cytoplasm of atp $4 \Delta$ and $s d h 2 \Delta$ and concomitantly increased in mitochondria of both mutants, whereas glycine is specifically reduced in $s d h 2 \Delta$ and normal for atp $4 \Delta$ mitochondria.

The gradient of nicotinic acid was dramatically altered in the mutants, with five to ten times reduced levels within mitochondria. The levels of the primary nicotinamide metabolite 1-methyl-nicotinamide were reduced in the cytoplasm of both analyzed mutants. In atp $4 \Delta$ mitochondria levels are $\sim 4$ times lower compared to WT. In contrast, in $s d h 2 \Delta$ mitochondria levels of 1-methyl-nicotinamide are increased by more than two-fold compared to WT, leading to opposing gradients between mitochondria and the cytoplasm. For the nucleic acid base uracil the levels were reduced by four times in the cytoplasm in atp $4 \Delta$ compared to WT. In contrast, for $s d h 2 \Delta$ the uracil ratio was shifted to the opposite direction with lower levels in mitochondria. Another very spectacular example of compartment-specific differential levels is pyrophosphate with reduced mitochondrial levels in both mutants, while in $s d h 2 \Delta$ cytoplasmic pyrophosphate was even more reduced compared to $\sim 6$ times increased cytoplasmic levels in atp $4 \Delta$ leading again to opposing gradients in both mutants. Interestingly, the alterations of TCA cycle intermediates were not only compartment-specific but

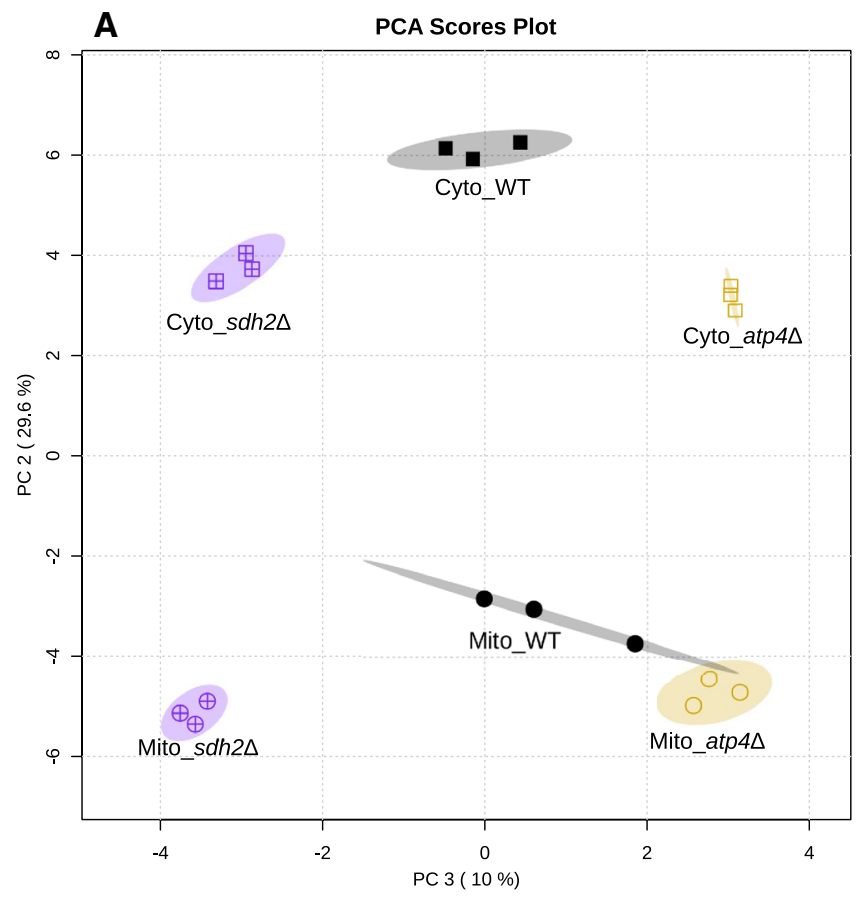

Fig. 4 a Principal component (PC) analysis plot and b its loading map. PC2 discriminates mitochondria (Mito) from the cytoplasm (Cyto), while PC3 discriminates atp $4 \Delta$ and $s d h 2 \Delta$ from each other and WT. The loading map displays how the individual metabolites

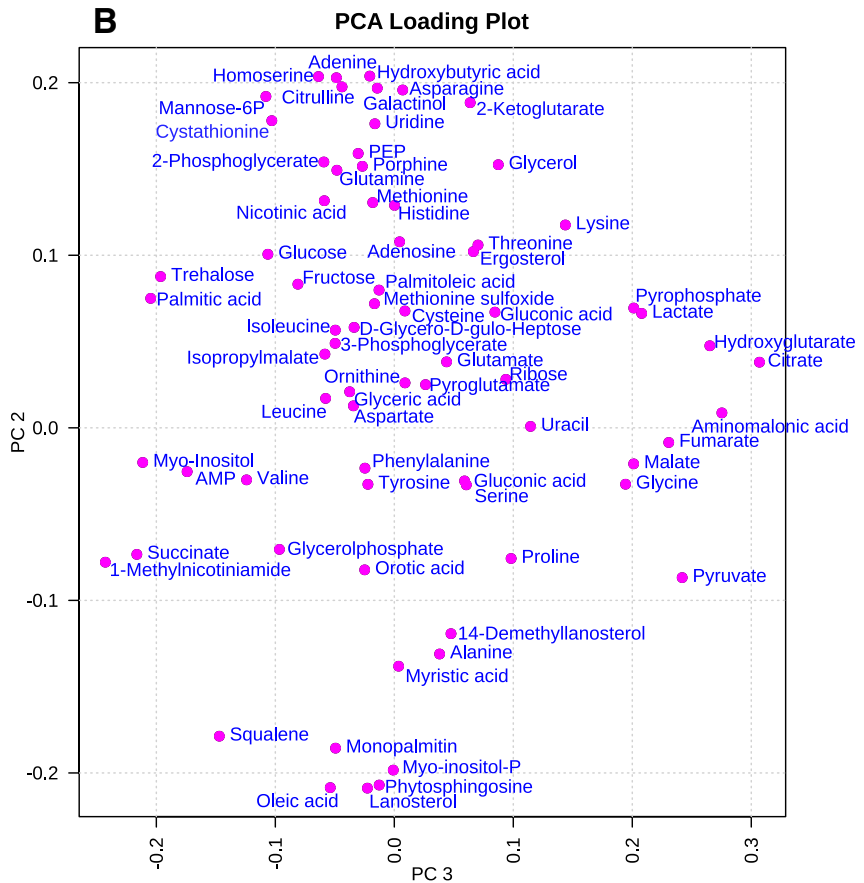

contribute to the discrimination. The bigger the absolute PC value of a metabolite is, the more it contributes to the discrimination in this PC 


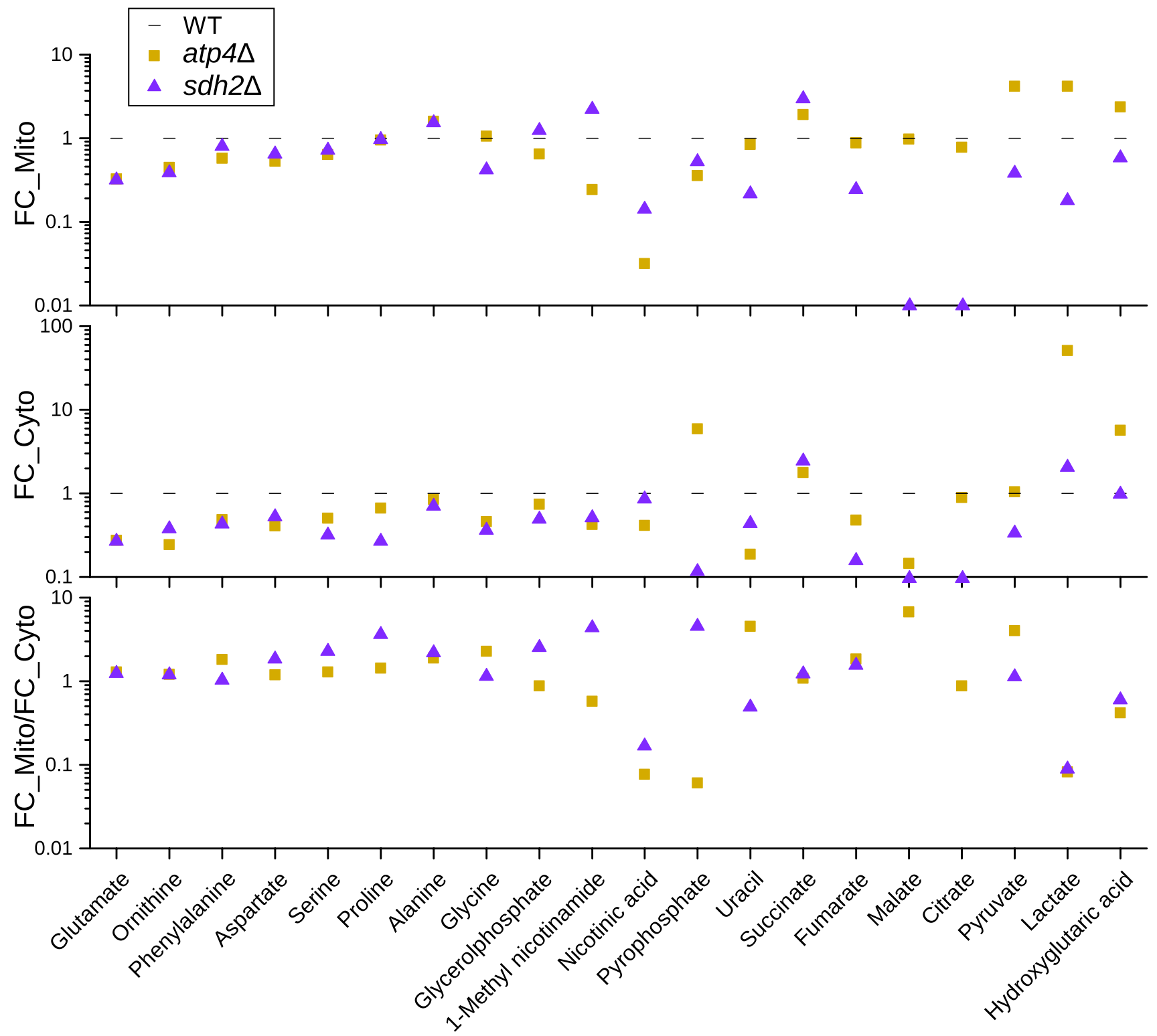

Fig. 5 Overview of fold changes (FC) of altered amino acids, TCA cycle intermediates and several other metabolites in mitochondria and cytoplasm of atp $4 \Delta$ and $s d h 2 \Delta$ cells. Not-detected metabolites are

also strain-specific. This will be discussed in the following paragraph.

\subsection{The TCA cycle is interrupted in sdh2 $\Delta$ but maintained in atp $4 \Delta$ cells}

Deletion of the succinate dehydrogenase gene $\mathrm{SDH} 2$ and the $\mathrm{FoF}_{1}$-ATP-synthase gene ATP4 causes a loss of function in respiratory complex II and complex V, resulting in impaired mitochondrial respiration. Consequently, TCA intermediates and related metabolites were affected not only in mitochondria but also in the cytoplasm. As shown in Fig. 5, succinate shown on the horizontal axes. FC_Mito/FC_Cyto indicates the compartment-specific metabolic alterations. The bigger the deviation of the value from 1 is, the more significant the difference is

is the single up-regulated metabolite with relation to the TCA cycle in both mutants and compartments. As succinate is oxidized to fumarate by succinate dehydrogenase, deletion of $\mathrm{SDH} 2$ resulted in an accumulation of succinate and a concomitant decrease of fumarate and malate in mitochondria. Although the $\mathrm{F}_{\mathrm{O}} \mathrm{F}_{1}$-ATP-synthase does not have a direct connection to succinate, its mutation also caused a significant accumulation of succinate. Since deletion of ATP4 interrupts ATP synthesis accompanied by accumulation of protons in the intermembrane space, elevated level of reduced coenzyme Q results in complex II inhibition and accumulation of succinate. As reviewed by Tretter et al. (2016), succinate 
is not only an intermediate in metabolism, but also plays an important role in signal transduction, ROS, hypoxia and tumorigenesis, which can induce massive metabolite alterations besides TCA cycle intermediates. Except for succinate, TCA cycle intermediates showed completely different regulation patterns between the two mutants. Fumarate, malate and citrate were down-regulated in both compartments of $s d h 2 \Delta$, while normal levels were detected in mitochondria of atp $4 \Delta$. Since deletion of $S D H 2$ interrupted the TCA cycle, its intermediates decreased overall, especially citrate and malate, which could not be detected either in mitochondria or in cytoplasm. Similar results were shown by Cardaci et al. (2015), demonstrating that SDH-deficient cells use pyruvate carboxylation to synthesize aspartate.

\section{Conclusion}

Since the metabolic response of mutations affecting the mitochondrial matrix metabolism can be buried in the pool of cytosolic metabolites, we established a compartmentspecific metabolic approach analyzing isolated mitochondria and their corresponding cytoplasmic fraction by applying GC-MS based metabolomics. Deletion of genes encoding the mitochondrial electron transport chain subunit Sdh2 and the FoF $_{1}$-ATPase subunit Atp4 resulted in massive metabolic alterations in both mitochondria and cytoplasm (Figs. 3, 5). The decrease of malate in the cytosol of atp $4 \Delta$ may be the result of accumulated mitochondrial NADH, triggering the cells to export reducing

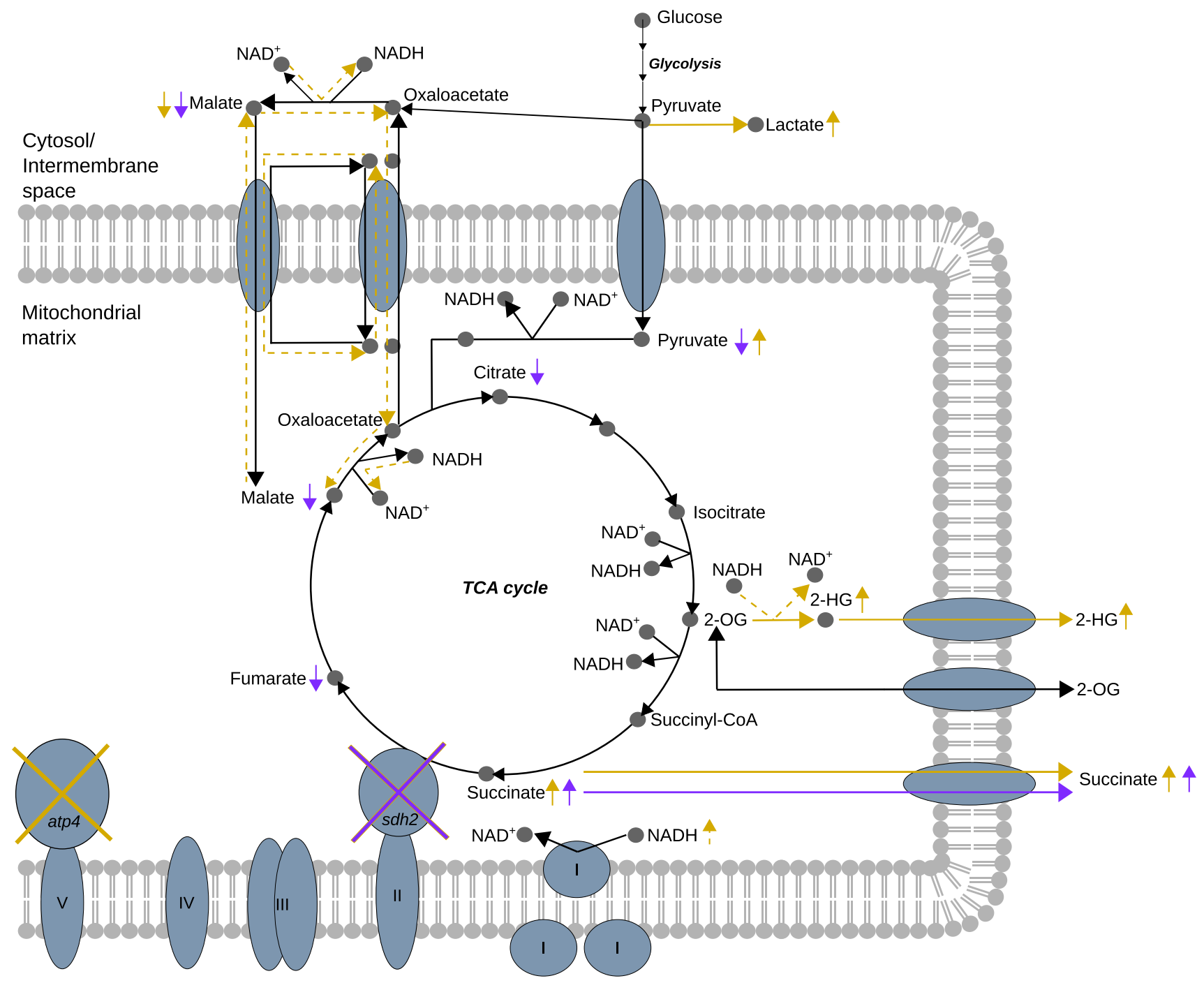

Fig. 6 Overview of metabolic alterations in mitochondria of $s d h 2 \Delta$ and atp $4 \Delta$ mutants. The relevant metabolic pathways are shown by solid black lines. The metabolic changes caused by the deletion of $S D H 2$ or ATP4 are displayed by either purple for $s d h 2 \Delta$ or deep yel- low for atp $4 \Delta$. The colored solid lines indicate the observed metabolic dysregulations and the dashed lines indicate the putative related metabolic changes (hypothesis of the authors) 
equivalents via reverse malate shuttle (Fig. 6). GOT1, as part of the malate-aspartate shuttle, has been described by Birsoy et al. (2015) to generate aspartate from oxaloacetate in the cytosol upon electron transport chain inhibition (complex I and III). Together with our results, it is tempting to speculate that cells can reverse this shuttle not only to produce aspartate, but also to export NADH. The exported malate is oxidized to oxaloacetate, generating cytosolic NADH, which can contribute to the reduction of pyruvate to lactate (highly accumulated in cytoplasm, see Fig. 5), indicating a metabolic state similar to anaerobic growth. In contrast to atp $4 \Delta$, the levels of cytoplasmic lactate are not significantly increased and mitochondrial lactate is even strongly reduced in $s d h 2 \Delta$ cells. Even though complex II activity is blocked in $s d h 2 \Delta$, reducing equivalents can be transferred to the electron transport chain via the NADH-dehydrogenases in the inner membrane (Marres et al. 1991; Luttik et al. 1998). This is supported by Kwon et al. (2015), showing a slightly decreased oxygen consumption rate in $s d h 2 \Delta$ cells and no oxygen consumption in atp $4 \Delta$ cells.

Besides lactate, 2-hydroxyglutarate was specifically elevated in the cytoplasm of atp $4 \Delta$ cells. Recently, the metabolic pathway involving 2-hydroxyglutarate for shuttling reducing equivalents from cytosol to mitochondria was described in yeast (Becker-Kettern et al. 2016). Considering the accumulation of reducing equivalents in atp $4 \Delta$ mitochondria, this import pathway may be employed reversely to export reducing equivalents from mitochondria to the cytosol. Reduction of mitochondrial 2-oxoglutarate generates 2-hydroxyglutarate, followed by transport to cytosol. Oxidation to 2-oxoglutarate by the mitochondrial retrograde response induced transhydrogenase Dld3 (Chelstowska et al. 1999), which concomitantly reduces pyruvate to lactate (Becker-Kettern et al. 2016), could also contribute to the high levels of cytosolic lactate in atp $4 \Delta$ cells. This function is in agreement with the proposed function for Dld3 to reduce the generation of NADH in respiratory deficient cells (Liu and Butow 2006). A model was generated in Fig. 6, describing the observed metabolic response in mitochondria of atp $4 \Delta$ and $s d h 2 \Delta$ cells. Taken together, this study shows that a compartment-specific metabolomics analysis is superior for the analysis and interpretation of pathological metabolic alterations and will be an important asset for future metabolic research.

Acknowledgements Work included in this study has also been performed in partial fulfillment of the requirements for the doctoral theses of D.P. and C.L. at the University of Freiburg. This work was supported by the European Research Council (ERC) Consolidator Grant 648235, the Excellence Initiative of the German federal and state governments (EXC 294, BIOSS; GSC-4, Spemann Graduate School), and Sonderforschungsbereich 1140 .
Author contributions NW and BK designed and supervised the project. DP performed the yeast culture, mitochondria isolation, metabolic profiling and data analysis, CL supported yeast culture, mitochondria isolation and performed mitochondrial integrity test, and SL supported metabolomics analysis and data interpretation. DP and CL prepared the figures and tables. BK and NW wrote the manuscript together with input from DP and CL. All authors discussed the results from the experiments and commented on the manuscript.

\section{Compliance with ethical standards}

Conflict of interest The authors declare no conflict of interest.

Research involving animal and human rights This article does not contain any studies with human participants or animals performed by any of the authors.

Open Access This article is distributed under the terms of the Creative Commons Attribution 4.0 International License (http://creativeco mmons.org/licenses/by/4.0/), which permits unrestricted use, distribution, and reproduction in any medium, provided you give appropriate credit to the original author(s) and the source, provide a link to the Creative Commons license, and indicate if changes were made.

\section{References}

Armitage, E. G., \& Barbas, C. (2014). Metabolomics in cancer biomarker discovery: Current trends and future perspectives. Journal of Pharmaceutical and Biomedical Analysis, 87, 1-11.

Asiago, V. M., Alvarado, L. Z., Shanaiah, N., Gowda, G. A. N., OwusuSarfo, K., Ballas, R. A., \& Raftery, D. (2010). Early detection of recurrent breast cancer using metabolite profiling. Cancer Research, 70, 8309-8318.

Baker Brachmann, C., Davies, A., Cost, G. J., Caputo, E., Li, J., Hieter, P., \& Boeke, J. D. (1998). Designer deletion strains derived from Saccharomyces cerevisiae S288C: A useful set of strains and plasmids for PCR-mediated gene disruption and other applications. Yeast, 14, 115-132.

Becker-Kettern, J., Paczia, N., Conrotte, J.-F., Kay, D. P., Guignard, C., Jung, P. P., \& Linster, C. L. (2016). Saccharomyces cerevisiae forms D-2-hydroxyglutarate and couples its degradation to D-lactate formation via a cytosolic transhydrogenase. The Journal of Biological Chemistry, 291, 6036-6058.

Birsoy, K., Wang, T., Chen, W. W., Freinkman, E., Abu-Remaileh, M., \& Sabatini, D. M. (2015). An essential role of the mitochondrial electron transport chain in cell proliferation is to enable aspartate synthesis. Cell, 162, 540-551.

Boldogh, I. R., \& Pon, L. A. (2007). Purification and subfractionation of mitochondria from the yeast Saccharomyces cerevisiae. Methods in Cell Biology, 80, 45-64.

Budczies, J., Pfitzner, B. M., Gyorffy, B., Winzer, K.-J., Radke, C., Dietel, M., et al. (2015). Glutamate enrichment as new diagnostic opportunity in breast cancer. International Journal of Cancer, $136,1619-1628$.

Bullinger, D., Neubauer, H., Fehm, T., Laufer, S., Gleiter, C. H., \& Kammerer, B. (2007). Metabolic signature of breast cancer cell line MCF-7: Profiling of modified nucleosides via LC-IT MS coupling. BMC Biochemistry, 8, 25.

Cardaci, S., Zheng, L., MacKay, G., van den Broek, N. J., MacKenzie, E. D., et al. (2015). Pyruvate carboxylation enables growth of 
SDH-deficient cells by supporting aspartate biosynthesis. Nature Cell Biology, 17, 1317-1326.

Chelstowska, A., Liu, Z., Jia, Y., Amberg, D., \& Butow, R. A. (1999). Signalling between mitochondria and the nucleus regulates the expression of a new D-lactate dehydrogenase activity in yeast. Yeast, 15, 1377-1391.

Chen, W. W., Freinkman, E., Wang, T., Birsoy, K., \& Sabatini, D. M. (2016). Absolute quantification of matrix metabolites reveals the dynamics of mitochondrial metabolism. Cell, 166, 1324-1337.

Corcelli, A., Saponetti, M. S., Zaccagnino, P., Lopalco, P., Mastrodonato, M., Liquori, G. E., \& Lorusso, M. (2010). Mitochondria isolated in nearly isotonic $\mathrm{KCl}$ buffer: Focus on cardiolipin and organelle morphology. Biochimica et Biophysica Acta, 1798, 681-687.

Fernández-Vizarra, E., Ferrín, G., Pérez-Martos, A., Fernández-Silva, P., Zeviani, M., \& Enríquez, J. A. (2010). Isolation of mitochondria for biogenetical studies: An update. Mitochondrion, 10, $253-262$

Fiehn, O. (2002). Metabolomics-The link between genotypes and phenotypes (pp. 155-171). Netherlands: Springer.

Fiehn, O. (2006). Metabolite profiling in Arabidopsis. Methods in Molecular Biology, 323, 439-447.

Fiehn, O., Kopka, J., Dormann, P., Altmann, T., Trethewey, R. N., \& Willmitzer, L. (2000). Metabolite profiling for plant functional genomics. Nature Biotechnology, 18, 1157-1161.

Frickenschmidt, A., Frohlich, H., Bullinger, D., Zell, A., Laufer, S., Gleiter, C. H., et al. (2008). Metabonomics in cancer diagnosis: Mass spectrometry-based profiling of urinary nucleosides from breast cancer patients. Biomarkers, 13, 435-449.

Gaude, E., \& Frezza, C. (2014). Defects in mitochondrial metabolism and cancer. Cancer \& Metabolism, 2, 10.

Goffeau, A., Barrell, B. G., Bussey, H., Davis, R. W., Dujon, B., Feldmann, H., et al. (1996). Life with 6000 genes. Science, 274, 546, 563-567.

Haan, C., \& Behrmann, I. (2007). A cost effective non-commercial ECL-solution for western blot detections yielding strong signals and low background. Journal of Immunological Methods, 318 , $11-19$.

Herrgard, M. J., Swainston, N., Dobson, P., Dunn, W. B., Arga, K. Y., Arvas, M., et al. (2008). A consensus yeast metabolic network reconstruction obtained from a community approach to systems biology. Nature Biotechnology, 26, 1155-1160.

Jain, M., Nilsson, R., Sharma, S., Madhusudhan, N., Kitami, T., Souza, A. L., et al. (2012). Metabolite profiling identifies a key role for glycine in rapid cancer cell proliferation. Science, 336, $1040-1044$.

Kind, T., Wohlgemuth, G., Lee, D. Y., Lu, Y., Palazoglu, M., Shahbaz, S., \& Fiehn, O. (2009). FiehnLib. Mass spectral and retention index libraries for metabolomics based on quadrupole and time-of-flight gas chromatography/mass spectrometry. Analytical Chemistry, 81, 10038-10048

Kopka, J., Schauer, N., Krueger, S., Birkemeyer, C., Usadel, B., Bergmüller, E., et al. (2005). GMD@CSB.DB: The Golm metabolome database. Bioinformatics, 21, 1635-1638.

Kwon, Y.-Y., Choi, K.-M., Cho, C., \& Lee, C.-K. (2015). Mitochondrial efficiency-dependent viability of Saccharomyces cerevisiae mutants carrying individual electron transport chain component deletions. Molecules and Cells, 38, 1054-1063.

Lasserre, J.-P., Dautant, A., Aiyar, R. S., Kucharczyk, R., Glatigny, A., Tribouillard-Tanvier, D., et al. (2015). Yeast as a system for modeling mitochondrial disease mechanisms and discovering therapies. Disease Models \& Mechanisms, 8, 509-526.

Liesenfeld, D. B., Habermann, N., Owen, R. W., Scalbert, A., \& Ulrich, C. M. (2013). Review of mass spectrometry-based metabolomics in cancer research. Cancer Epidemiology, Biomarkers \& Prevention, 22, 2182-2201.
Liu, Z., \& Butow, R. A. (2006). Mitochondrial retrograde signaling. Annual Review of Genetics, 40, 159-185.

Luttik, M. A. H., Overkamp, K. M., Kötter, P., de Vries, S., van Dijken, J. P., \& Pronk, J. T. (1998). The Saccharomyces cerevisiae NDE1 and NDE2 genes encode separate mitochondrial NADH dehydrogenases catalyzing the oxidation of cytosolic NADH. Journal of Biological Chemistry, 273, 24529-24534.

Marres, C. A. M., Vries, S., \& Grivell, L. A. (1991). Isolation and inactivation of the nuclear gene encoding the rotenone-insensitive internal NADH: Ubiquinone oxidoreductase of mitochondria from Saccharomyces cerevisiae. European Journal of Biochemistry, 195, 857-862.

Matuszczyk, J.-C., Teleki, A., Pfizenmaier, J., \& Takors, R. (2015). Compartment-specific metabolomics for $\mathrm{CHO}$ reveals that ATP pools in mitochondria are much lower than in cytosol. Biotechnology Journal, 10, 1639-1650.

Meisinger, C., Pfanner, N., \& Truscott, K. N. (2006). Isolation of yeast mitochondria. Methods in Molecular Biology, 313, 33-39.

Mogensen, M., Sahlin, K., Fernström, M., Glintborg, D., Vind, B. F., Beck-Nielsen, H., \& Højlund, K. (2007). Mitochondrial respiration is decreased in skeletal muscle of patients with type 2 diabetes. Diabetes, 56, 1592-1599.

Morgenstern, M., Stiller, S. B., Lübbert, P., Peikert, C. D., Dannenmaier, S., Drepper, F., et al. (2017). Definition of a high-confidence mitochondrial proteome at quantitative scale. Cell Reports, 19, 2836-2852.

Nunnari, J., \& Suomalainen, A. (2012). Mitochondria. In sickness and in health. Cell, 148, 1145-1159.

Paglia, G., Stocchero, M., Cacciatore, S., Lai, S., Angel, P., Alam, M. T., et al. (2016). Unbiased metabolomic investigation of Alzheimer's disease brain points to dysregulation of mitochondrial aspartate metabolism. Journal of Proteome Research, 15, 608-618.

Pan, D., Kather, M., Willmann, L., Schlimpert, M., Bauer, C., Lagies, S., et al. (2016). Metabolic response to XD14 treatment in human breast cancer cell line MCF-7. International Journal of Molecular Sciences, 17, 1772.

Roede, J. R., Park, Y., Li, S., Strobel, F. H., \& Jones, D. P. (2012). Detailed mitochondrial phenotyping by high resolution metabolomics. PLoS ONE, 7, e33020.

Ross-Inta, C., Tsai, C.-Y., \& Giulivi, C. (2008). The mitochondrial pool of free amino acids reflects the composition of mitochondrial DNA-encoded proteins: Indication of a post-translational quality control for protein synthesis. Bioscience Reports, 28, 239-249.

Sana, T. R., Gordon, D. B., Fischer, S. M., Tichy, S. E., Kitagawa, N., Lai, C., et al. (2013). Global mass spectrometry based metabolomics profiling of erythrocytes infected with Plasmodium falciparum. PLoS ONE, 8, e60840.

Schapira, A. H. V. (2012). Mitochondrial diseases. The Lancet, 379, $1825-1834$.

Shen, J., Yan, L., Liu, S., Ambrosone, C. B., \& Zhao, H. (2013). Plasma metabolomic profiles in breast cancer patients and healthy controls: By race and tumor receptor subtypes. Translational Oncology, 6, 757-765.

Siess, E. A., Brocks, D. G., \& Wieland, O. H. (1978). Distribution of metabolites between the cytosolic and mitochondrial compartments of hepatocytes isolated from fed rats. Hoppe-Seyler's Zeitschrift fur physiologische Chemie, 359, 785-798.

Stefely, J. A., Kwiecien, N. W., Freiberger, E. C., Richards, A. L., Jochem, A., Rush, M. J. P., et al. (2016). Mitochondrial protein functions elucidated by multi-omic mass spectrometry profiling. Nature Biotechnology, 34, 1191-1197.

Styczynski, M. P., Moxley, J. F., Tong, L. V., Walther, J. L., Jensen, K. L., \& Stephanopoulos, G. N. (2007). Systematic identification of conserved metabolites in GC/MS data for metabolomics and biomarker discovery. Analytical Chemistry, 79, 966-973. 
Tretter, L., Patocs, A., \& Chinopoulos, C. (2016). Succinate, an intermediate in metabolism, signal transduction, ROS, hypoxia, and tumorigenesis. Biochimica et Biophysica Acta, 1857, 1086-1101.

van der Bliek, A. M., Sedensky, M. M., \& Morgan, P. G. (2017). Cell biology of the mitochondrion. Genetics, 207, 843-871.

van Vranken, J. G., \& Rutter, J. (2016). The whole (cell) is less than the sum of its parts. Cell, 166, 1078-1079.

Wiedemann, N., \& Pfanner, N. (2017). Mitochondrial machineries for protein import and assembly. Annual Review of Biochemistry, 86, 685-714.

Willmann, L., Schlimpert, M., Halbach, S., Erbes, T., Stickeler, E., \& Kammerer, B. (2015). Metabolic profiling of breast cancer:
Differences in central metabolism between subtypes of breast cancer cell lines. Journal of Chromatography B, 1000, 95-104.

Willmann, L., Schlimpert, M., Hirschfeld, M., Erbes, T., Neubauer, H., Stickeler, E., \& Kammerer, B. (2016). Alterations of the exoand endometabolite profiles in breast cancer cell lines: A mass spectrometry-based metabolomics approach. Analytica Chimica Acta, 925, 34-42.

Xia, J., Sinelnikov, I. V., Han, B., \& Wishart, D. S. (2015). MetaboAnalyst 3.0-Making metabolomics more meaningful. Nucleic Acids Research, 43, W251-W257. 